Z Epileptol 2018 $\cdot 31: 240-244$ https://doi.org/10.1007/s10309-018-0212-z Online publiziert: 26 . September 2018 (c) Der/die Autor(en) 2018

CrossMark

Markus Leitinger - Eugen Trinka

Universitätsklinik für Neurologie, Christian Doppler Klinik, Paracelsus Medizinische Universität Salzburg, Salzburg, Österreich

\title{
Nonkonvulsiver Status epilepticus
}

\section{Diagnosekriterien}

\section{Literaturüberblick}

ticus (NCSE) beinhaltet gemäß der Definition und Klassifikation der Internationalen Liga gegen Epilepsie (ILAE) 2015 keine „prominenten motorischen Phänomene", jedoch können subtile klinische Zeichen, wie beispielsweise periorbitale oder periorale Zuckungen, ein konjugierter Herdblick oder ein Hippus pupillae, auftreten. Ohne diese beruht die Diagnostik auf der Auswertung des Elektroenzephalogramms. Dieser Artikel stellt die Evidenzlage für Diagnosekriterien des NCSE und deren konkrete Anwendung im klinischen Alltag dar.

In der neuen Definition und Klassifikation des Status epilepticus (SE) der Internationalen Liga Gegen Epilepsie (ILAE) 2015 wird zwischen SE mit ,prominenten motorischen Phänomenen" (einschließlich bilateral tonisch klonischem SE, fokal motorischem SE, tonischem SE, etc.) einerseits und dem nichtkonvulsiven SE (NCSE) mit allenfalls subtilen motorischen Phänomenen andererseits unterschieden [1]. Der nichtkonvulsive Status epilepticus (NCSE) tritt klinisch als qualitative oder quantitative Bewusstseinsstörung, Verhaltensabnormität, vegetative Reaktion oder als rein subjektive Wahrnehmung, i. e. Aura, in Erscheinung [2]. Da diese Symptomatik unspezifisch ist und beispielsweise auch durch akute zerebrale Ischämien hervorgerufen werden kann, wird im Regelfall eine EEGAbleitung zur Diagnosestellung bzw. -sicherung herangezogen.
Es bestehen 2 grundsätzlich verschiedene Wege, das EEG auszuwerten. Im exemplarischen Weg werden aussagekräftige Beispiele gesammelt, die als Vorlage für zukünftige EEG-Befundungen herangezogen werden. In einem systematischen Review wurden 123 Artikel mit EEG-Muster des NCSE gefunden [3]. Dem Vorteil der Anschaulichkeit stehen die Nachteile gegenüber, dass die gesammelten Beispiele mehrdeutig sein oder nur die am stärksten ausgeprägten Muster zeigen können [3]. Alternativ hierzu werden im operationalisierten Zugang Kriterien entwickelt, die eine objektive Auswertung des EEGs und die Reproduzierbarkeit des Ergebnisses sicherstellen sollen. Kriterien für epileptiforme Entladungen, rhythmische Aktivität, Evolutionsmuster sowie Ansprechen auf intravenöse Antiepileptika zur Diagnose nichtkonvulsiver Anfälle wurden von Young et al. formuliert und von Chong und Hirsch präzisiert $[4,5]$. Die Differenzierung zwischen Patienten mit bzw. ohne epileptische Enzephalopathie geht auf Kaplan et al. zurück [6]. Im Rahmen des London-Innsbruck Colloquium on Status epilepticus and Acute seizures, das 2013 in Salzburg stattfand, wurde von einer Expertengruppe ein Set an Kriterien für den "möglichen NCSE“ und den „NCSE“ zusammengestellt („Salzburg-Kriterien“) [7]. Dieser Kriteriensatz wurde, mit konkreten Definitionen und Anwendungsregeln ausgestattet, retrospektiv an 3 Zentren (Dänemark: Aarhus Universität, Aarhus, Epilepsiezentrum Filadelfia, Dianalund; Österreich: Salzburg) evaluiert, wobei es sich um die bisher einzige Evaluierungsstudie handelt [9]. In der klinischen Anwendung wurde ein Diagnoseschema entwickelt, das die klinische Komponente der Diagnosestellung definiert und die Reduktion der Falsch-Positiven zum Ziel hat [8].

\section{Konkrete Anwendung}

\section{Allgemeines}

Die Diagnose des NCSE setzt sich aus klinischen und EEG-Kriterien zusammen (๑ Abb. 1 gemäß [11]; [8]). Die nachfolgenden EEG-Kriterien müssen in ihrer ausgeprägtesten Form für jeweils $10 \mathrm{~s}$ erfüllt werden, wiewohl die EEG-Ableitung zur Gänze bzw. über weite Strecken pathologisch ist. Zur Diagnosestellung des NCSE sollen „die $10 \mathrm{~s}$ mit der stärksten Ausprägung" der Kriterien verwendet werden. Eine Verlängerung auf 30 bzw. 60 s bewirkte lediglich eine moderate Reduktion der Sensitivität bei minimalem Zuwachs an Spezifität [9].

\section{》) Die Diagnose des NCSE setzt sich aus klinischen und EEG- \\ Kriterien zusammen}

Die Diagnoseoptionen sollen nach Meinung der Autoren aus „NCSE“ oder „kein NCSE“ bestehen. Der Ausdruck „möglicher NCSE“ ist in mehrerer Hinsicht problematisch. Als wichtigstes Argument ist anzuführen, dass der Elektrophysiologe in Kenntnis der Anamnese, des neurologischen Befundes und der Zusatzuntersuchungen sowie der Reagibilität des EEGs und des Patienten während der Ab- 


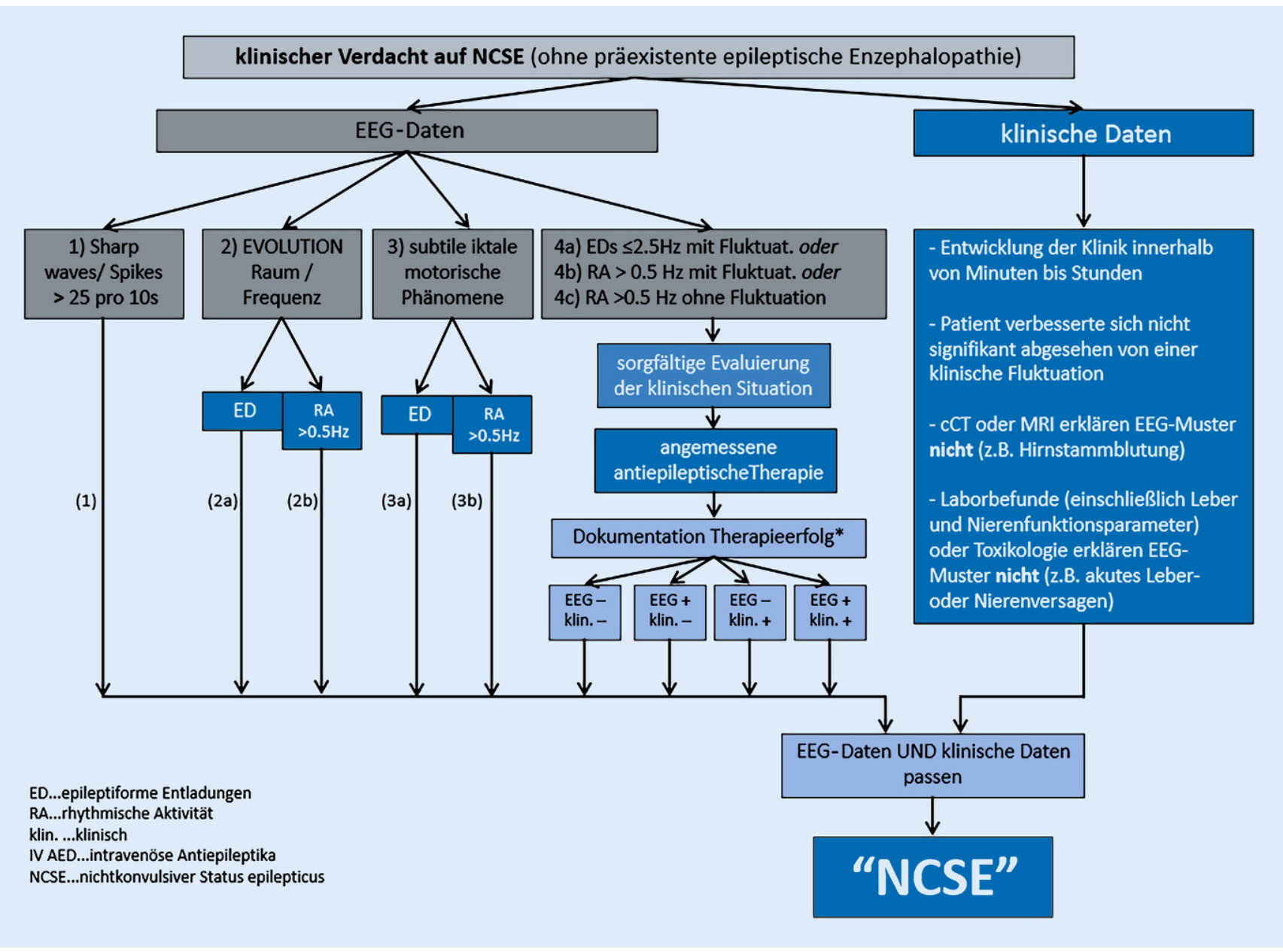

Abb. 1 A Diagnoseschema "modifizierte Salzburg-Kriterien des NCSE“ gemäß [11]:ED Epileptiforme Entladungen, RA Rhythmische Aktivität, klin klinisch, NCSE Nichtkonvulsiver Status epilepticus, * auf intravenöse Antiepileptika (iv AED)

leitung das Maximum an Information zur Verfügung hat, um einen NCSE zu diagnostizieren oder zu verwerfen. „Möglicher NCSE“ wird vielmehr vom Zuweiser verwendet, der rasch das Vertrauen in die Elektrophysiologie verlieren kann, wenn man ihm die Zuweisungsdiagnose als Ergebnis zurückreicht.

\section{Die EEG-Kriterien}

\section{Kriterium 1: epileptiforme Aktivität über 2,5/s}

Die Messung der epileptiformen Graphoelemente in einer 10-s-Epoche wird als zuverlässiger angesehen als die Messung der Graphoelemente pro Sekunde oder die Berechnung der Frequenz aus dem Kehrwert der Periodendauer, insbesondere wenn epileptiforme Aktivität mit variabler Periodendauer auftritt (somit „epileptiforme Aktivität über
25/10 s"). Der Wert 25/10 s ist ein Stück weit willkürlich, ist jedoch sensitiver bezogen auf 30/10 s-Phänomene, wie z.B. Absencenstatus, und andererseits spezifisch bezogen auf 10 bis 15/10 s lateralisierte periodische Entladungen (LPDs). Jede Veränderung des Grenzwertes kann als eine Verschiebung entlang der sog. ROC(„Receiver Operating Characteristics“)-Kurve verstanden werden, wobei eine Erhöhung einen Gewinn an Spezifität und einen Verlust an Sensitivität bewirkt und vice versa. Die Entscheidung, welcher Grenzwert an einem Zentrum verwendet wird, kann nach Abwägung der Für und Wider festgelegt werden. Für die medizinische Gemeinschaft ist es jedoch von Vorteil, wenn eine große Menge an Erfahrung einheitlich gesammelt wird und auf diesen Erkenntnissen aufbauend die Strategie optimiert wird.

\section{Kriterium 2: subtile klinische Phänomene}

Subtile klinische Phänomene können mit epileptiformer Aktivität oder rhythmischer Aktivität einhergehen und meinen beispielsweise periorbitale oder periorale Zuckungen, subtile Zuckungen an den Extremitäten, Hippus pupillae oder forcierte konjugierte Blickwendung. Diese Phänomene treten nicht ausschließlich beim nichtkonvulsiven Status epilepticus auf, beispielsweise medikamenteninduzierte Myoklonien oder ein ischämisch bedingter Herdblick. Nach Meinung der Autoren soll deshalb ein Akut-EEG zur Diagnosestellung des NCSE erfolgen, andererseits kann bei klarem und eindeutigem passendem anamnestischem und klinischem Kontext (e.g. bilateral tonisch klonischer Status für $40 \mathrm{~min}$ und Übergehen in subtile Zuckungen des rechten Daumens) die Diagnose 
NCSE gestellt und noch vor Eintreffen des EEGs eine Akuttherapie eingeleitet werden. Demgegenüber ist das alleinige Auftreten von lateralisierten periodischen Entladungen (LPDs) ohne unmittelbares klinisches Korrelat nicht als SE einzustufen [9].

\section{Kriterium 3: Evolutionsmuster}

Evolutionsmuster treten in der Domäne Frequenz als Dekrement- oder Inkrementreaktion auf [9]. Gemäß Amerikanischer Klinisch Neurophysiologischer Gesellschaft (ACNS) sind auch die räumliche Ausbreitung und die Veränderung der Morphologie operationalisiert, um ein Maximum an einheitlicher Herangehensweise $\mathrm{zu}$ erzielen [10]. Von besonderer Bedeutung ist, dass die ACNSKriterien lediglich Graphoelemente und deren Verteilung oder Häufigkeit definieren, jedoch per se keine Statuskriterien darstellen [10].

\section{Kriterium 4: Fluktuation und rhythmische Aktivität ohne Evolution}

Während die Kriterien 1 bis 3 in einem normalen EEG wahrscheinlich niemals zu erwarten sind, kann fluktuierende epileptiforme Aktivität von 2,5/s oder weniger bzw. rhythmische Aktivität mit oder ohne Fluktuation sehr wohl bei pathologischen EEGs ohne NCSE vorkommen. Deshalb ist insbesondere bei Kriterium 4 die Einbeziehung der klinischen und paraklinischen Information von besonderer Bedeutung, da akutes Nieren- oder Leberversagen, Intoxikationen mit Lithium etc. oder ein Hirnstamminfarkt statusähnliche Muster bewirken können. Weitere Beispiele sind Sepsis oder SchädelHirn-Trauma.

\section{Kriterium 5: Ansprechen auf intravenöse Antiepileptika}

Manche Autoren definieren das Ansprechen sowohl klinisch als auch im EEG auf intravenöse Antiepileptika (ivAED) als „NCSE“, während das alleinige Ansprechen im EEG als „möglicher NCSE“ eingestuft wird [7]. Das fehlende Ansprechen klinisch und im EEG lässt hierbei keine NCSE-Diagnose zu [7]. Dem ist entgegenzuhalten, dass es diesen Regeln folgend gar keine therapierefraktä-

Z Epileptol 2018·31:240-244 https://doi.org/10.1007/s10309-018-0212-z

(c) Der/die Autor(en) 2018

\section{Leitinger · E. Trinka}

\section{Nonkonvulsiver Status epilepticus. Diagnosekriterien}

\section{Zusammenfassung}

Hintergrund. Der nichtkonvulsive Status epilepticus (NCSE) ist mit einem schlechten Outcome assoziiert und stellt darüber hinaus im klinischen Alltag eine diagnostische Herausforderung dar.

Fragestellung. Es existieren mehrere Sets an diagnostischen Kriterien. Was unterscheidet sie und welche sind evaluiert? Welches Konzept kann nach heutigem Stand des Wissens empfohlen werden?

Material und Methode. Es wird ein Überblick über die bisherige Literatur und die strukturierte Vorgehensweise zur Diagnosestellung des NCSE gegeben.

Ergebnisse. Es existiert nur eine einzige Evaluierung diagnostischer Kriterien des NCSE, i. e. die "Salzburg-Kriterien des NCSE", diese wurde retrospektiv multizentrisch durchgeführt. Die Diagnosestellung basiert auf der Implementierung klinischer und EEG-Daten. Die weitere Einbeziehung paraklinischer Information wie zerebrale Bildgebung, Notfalllabor und Toxikologielabor ist unerlässlich.

Schussfolgerung. Mit den derzeit verfügbaren Kriterien lässt sich die große Mehrheit an Patienten mit NCSE-Verdacht effektiv und effizient abklären. Im Einzelfall muss berücksichtigt werden, dass die Kriterien falsch negativ bzw. falsch positiv sein können.

Schlüsselwörter

Status epilepticus - Non convulsive - Criteria . Diagnose $\cdot$ EEG

\section{Nonconvulsive status epilepticus. Diagnostic criteria}

\section{Abstract}

Background. Nonconvulsive status epilepticus (NCSE) is associated with a poor outcome and is furthermore a diagnostic challenge in routine clinical work.

Objective. Several sets of diagnostic criteria exist. What is the difference between them and which ones have been evaluated? Which concept can be recommended in the light of current knowledge?

Material and methods. A survey on the current literature is provided and a structured approach for diagnosing NCSE is given. Results. Only one study on evaluation of the diagnostic criteria for NCSE exists, i.e. the "Salzburg criteria for NCSE", which were investigated retrospectively in a multicenter study. The diagnosis of NCSE is based on clinical and EEG data. The additional implementation of paraclinical information, such as cerebral imaging, emergency and toxicology laboratories is mandatory. Conclusion. Currently available criteria enable efficient and effective management of the majority of patients suspected of having NCSE; however, in individual cases it has to be taken into consideration that the criteria can be falsely positive or falsely negative.

\section{Keywords}

Status epilepticus - Nonconvulsive · Criteria . Diagnosis · EEG ren NCSE geben könnte. Des Weiteren ist nicht definiert, wie viele Medikamente in welcher Dosis an ivAED zu verabreichen sind, um die Beurteilung durchführen zu können. Somit wird empfohlen, jedwede Konstellation an Ansprechen potenziell zur Diagnose zuzulassen, jedoch dieses Phänomen als Mosaikstein in der klinischen Gesamtsituation zu interpretieren. Im Einzelfall kann eine probatorische Gabe von ivAED in Erwägung gezogen werden, jedoch darf wie angeführt bei fehlendem Ansprechen kein NCSE ausgeschlossen werden. An- dererseits darf bei Sistieren bifrontaler Delta-Aktivität wie bei Enzephalopathie auf ivAED nicht zwangsläufig auf einen NCSE geschlossen werden, da sich enzephalopathieassoziierte EEG-Muster sehr gut durch z.B. Benzodiazepine reduzieren lassen.

\section{Klinische Kriterien}

\section{Klinisches Zustandsbild}

Der Übergang vom gesunden in den jetzigen kranken Zustand erfolgte im Laufe von Minuten bis Stunden, somit sind Se- 
kunden bzw. Tage und Wochen wenig für NCSE qualifizierend. Das Zustandsbild soll sich in den letzten Minuten oder Stunden nicht wesentlich geändert haben, abgesehen von dem für NCSE typischen Fluktuieren. Dies soll beispielsweise die zunehmende Erholung nach einem bilateral tonisch klonischen Anfall als gering wahrscheinlich für NCSE einstufen. Ein pathologisches EEG ohne jedwede neurologische Symptomatik kann nicht als NCSE diagnostiziert werden, sondern vielmehr als pathologisches Muster mit Hinweis auf erhöhte zerebrale Erregungsbereitschaft. Allerdings kann die Symptomatik des NCSE sehr subtil sein, beispielsweise nur dem Patienten als „anders als sonst“ oder im Rahmen einer elaborierten neuropsychologischen Testung auffallen.

\section{Paraklinische Information}

Bei jeder wesentlichen Funktionsstörung des Gehirns ist ein Korrelat im EEG zu erwarten. Manche EEG-Muster repräsentieren somit die primäre Schädigung und nicht zwangsläufig einen zusätzlich ausgelösten Status epilepticus. Beispielsweise können Hirnstammblutungen eine weitstreckige bilaterale Delta-Aktivität hervorrufen, ohne dass dies als NCSE einzustufen ist, obwohl der Patient eine schwere Bewusstseinsstörung aufweisen kann. Analoges gilt für epileptiforme Potenziale, hervorgerufen durch Intoxikationen mit Lithium oder akutem Nierenoder Leberversagen.

\section{) Paraklinische Information (cCT, MRI, Toxikologie, Akutlabor) reduziert falsch positive Ergebnisse}

Der standardisierte Zugang zur Evaluierung eines NCSE-suspekten EEGs schließt die Beurteilung von Anamnese, zerebraler Bildgebung, Notfalllabor und Toxikologielabor unabdingbar mit ein.

\section{Zu erwartende Testperformance}

Die in der retrospektiven Evaluierung erzielte Sensitivität betrug $98 \%$ bei einer Spezifität von $90 \%$, der positive prädik- tive Wert lag bei $84 \%$, der negative prädiktive Wert bei 99\% [9]. Die Gesamtgenauigkeit war mit 92,5\% gegeben. Das die Interraterreliabilität repräsentierende Kappa lag bei 0,87 für die Anwendung der Salzburg-Kriterien in der Gesamtgruppe der Studie (Validierungs- und Kontrollgruppe; [9]).

\section{Caveats}

Von besonderer Wichtigkeit ist, dass es sich bei den Diagnosekriterien immer um Arbeitshypothesen handelt. Sie erlauben es, bei einem Großteil der Patienten rasch und verlässlich zur Diagnose zu gelangen. Allerdings können die Kriterien falsch negativ oder falsch positiv sein. Die Würdigung des gesamten klinischen und paraklinischen Kontexts ist unerlässlich. Die Kriterien geben keinen Hinweis darauf, wie aggressiv die Therapie ausgeführt werden soll.

Limitationen. An zukünftigen Aufgaben ist festzulegen, was unter einem „Ansprechen im EEG“ oder einem „klinischen Ansprechen" auf ivAED zu verstehen ist, um eine homogene Anwendung zu ermöglichen. Ein Vorschlag hierzu wurde bereits von der Salzburger Arbeitsgruppe vorgelegt, jedoch noch nicht evaluiert [8]. Ungeklärt ist des Weiteren die Frage nach dem optimalen Follow-up im EEG bzw. der Indikation zum Langzeit-EEG.

Zusammenfassung. Bislang existiert nur eine einzige Evaluierung von Diagnosekriterien des NCSE, diese wurde retrospektiv multizentrisch durchgeführt. Das aktuellste Diagnoseschema inkludiert klar definierte EEG- und klinische Konstellationen. Im Einzelfall ist zu berücksichtigen, dass auch evaluierte Diagnosekriterien falsch positiv oder falsch negativ sein können.

\section{Fazit für die Praxis}

- Das EEG ist die wichtigste paraklinische Untersuchung zur Diagnose des NCSE, bedarf aber konkreter Diagnosekriterien und weiterer paraklinischer Untersuchungen wie zerebraler Bildgebung, Labor und To- xikologie zur Abgrenzung von falsch positiven Ergebnissen.

- EEG-Diagnosekriterien des NCSE müssen standardisiert erhoben werden. Die Amerikanische Klinische Neurophysiologische Gesellschaft (ACNS) stellt Diagnosekriterien für EEG-Graphoelemente und -Muster bereit, die in einem weiteren Schritt mittels separater EEG-Diagnosekriterien des NCSE ausgewertet werden.

- Subtile klinische Phänomene, e.g. periorale Zuckungen, sollten den klinischen Verdacht auf einen NCSE erheben und bei eindeutig passender Anamnese und Klinik eine Akuttherapie in Erwägung ziehen lassen.

- Die Salzburg-Kriterien sind die bislang einzigen (retrospektiv) evaluierten Kriterien des NCSE mit sehr hoher Sensitivität und hoher Spezifität.

- Für einige wichtige Definitionen fehlt der internationale Konsens, z. B. „wann gilt ein NCSE als durchbrochen?".

\section{Korrespondenzadresse}

\section{Dr. Markus Leitinger}

Universitätsklinik für Neurologie, Christian

Doppler Klinik, Paracelsus Medizinische

Universität Salzburg

5020 Salzburg, Österreich

ma.leitinger@salk.at

Funding. Open access funding provided by Paracelsus Medical University.

\section{Einhaltung ethischer Richtlinien}

Interessenkonflikt. M. Leitinger und E. Trinka geben an, dass kein Interessenkonflikt besteht.

Dieser Beitrag beinhaltet keine von den Autoren durchgeführten Studien an Menschen oder Tieren.

Open Access. Dieser Artikel wird unter der Creative Commons Namensnennung 4.0 International Lizenz (http://creativecommons.org/licenses/by/4.0/deed. de) veröffentlicht, welche die Nutzung, Vervielfältigung, Bearbeitung, Verbreitung und Wiedergabe in jeglichem Medium und Format erlaubt, sofern Sie den/die ursprünglichen Autor(en) und die Quelle ordnungsgemäßnennen, einen Linkzur Creative Commons Lizenz beifügen und angeben, ob Änderungen vorgenommen wurden. 


\section{Leitthema}

\section{Literatur}

1. Trinka $E$, Cock $H$, Hesdorffer D, Rossetti AO, Scheffer IE, Shinnar S, Shorvon S, Lowenstein DH (2015) A definition and classification of status epilepticus-Report of the ILAE Task Force on Classification of Status Epilepticus. Epilepsia 56(10):1515-1523. https://doi.org/10.1111/epi. 13121

2. Drislane FW (2000) Presentation, evaluation, and treatment of nonconvulsive status epilepticus. Epilepsy Behav 1:301-314

3. Sutter R, Kaplan PW (2012) Electroencephalographic criteria for nonconvulsive status epilepticus: synopsis and comprehensive survey. Epilepsia 3:1-51. https://doi.org/10.1111/j.1528-1167. 2012.03593.x

4. Young GB, Jordan KG, Doig GS (1996) An assessment of nonconvulsive seizures in the intensive care unit using continuous EEG monitoring: an investigation of variables associated with mortality. Neurology 47(1):83-89

5. Chong DJ, Hirsch LJ (2005) Which EEG patterns warrant treatment in the critically ill? Reviewing the evidence for treatment of periodic epileptiform discharges and related patterns. J Clin Neurophysiol 22(2):79-91

6. Kaplan PW (2007) EEG criteria for nonconvulsive status epilepticus. Epilepsia 48(Suppl 8):39-41

7. Beniczky S, Hirsch LJ, Kaplan PW, Pressler R, Bauer G, Aurlien H, Brøgger JC, Trinka E (2013) Unified EEG terminology and criteria for nonconvulsive status epilepticus. Epilepsia 54(Suppl 6):28-29. https:// doi.org/10.1111/epi.12270

8. Leitinger M, Beniczky S, Rohracher A, Gardella E, Kalss G, Qerama E, Höfler J, Hess Lindberg-Larsen A, Kuchukhidze G, Dobesberger J, Langthaler PB, Trinka E (2015) Salzburg consensus criteria for non-convulsive status epilepticus-approach to clinical application. Epilepsy Behav 49:158-163. https://doi.org/10.1016/j.yebeh.2015.05.007

9. Leitinger M, Trinka E, Gardella E, Rohracher A, Kalss G, Qerama E, Höfler J, Hess A, Zimmermann $G$, Kuchukhidze G, Dobesberger J, Langthaler PB, Beniczky S (2016) Diagnostic accuracy of the Salzburg EEG criteria for non-convulsive status epilepticus: a retrospective study. Lancet Neurol 15(10):1054-1062. https://doi.org/10. 1016/S1474-4422(16)30137-5

10. Hirsch LJ, LaRoche SM, Gaspard N, Gerard E, Svoronos A, Herman ST, Mani R, Arif $H$, Jette $N$, Minazad $Y$, Kerrigan JF, Vespa $P$, Hantus $S$, Claassen J, Young GB, So E, Kaplan PW, Nuwer MR, Fountain NB, Drislane FW (2013) American clinical neurophysiology society's standardized critical care EEG terminology: 2012 version. JClin Neurophysiol 30(1):1-27. https://doi.org/10 1097/WNP.0b013e3182784729

11. Trinka $E$, Leitinger $M$ (2015) Which EEG patterns in coma are nonconvulsive status epilepticus? Epilepsy Behav 49:203-222. https://doi.org/10. 1016/j.yebeh.2015.05.005 